\title{
Terminologia e organização do conhecimento: linguagens, vocabulários e sistemas
}

Terminology and Knowledge Organization: Languages, Vocabularies and Systems

\author{
Bruno Almeida* \\ brunoalmeida@fcsh.unl.pt \\ https://orcid.org/0000-0002-5777-5574
}

\section{Resumo}

Este artigo parte do pressuposto de que as ferramentas utilizadas para a organização do conhecimento (tesauros, esquemas de classificação, etc.) podem ser entendidas como recursos terminológicos. Abordamos as relações entre terminologia e organização do conhecimento, assumindo um ponto de vista baseado na bidimensionalidade (linguística e conceptual) da terminologia enquanto disciplina. Em seguida, propomos uma análise dos conceitos subjacentes às designações "linguagem documental", "vocabulário controlado" e "sistema de organização do conhecimento" nos textos de especialidade. Terminamos com a descrição do SKOS (Simple Knowledge Organization System), um modelo para a representação de sistemas de organização do conhecimento na web semântica, o qual é avaliado em termos da sua capacidade de modelizar recursos terminológicos de acordo com a abordagem bidimensional à terminologia e os principais elementos da norma ISO 1087.

Palavras-chave: Sistemas de organização de conhecimento; Vocabulários controlados; Linguagens documentais; Recursos terminológicos; SKOS.

\begin{abstract}
This article assumes that the tools used for knowledge organization purposes (e.g., thesauri, classification schemes...) may be understood as terminological resources. We investigate the relationship between terminology and knowledge organization from the doubledimension perspective (both linguistic and conceptual) of terminology as a field of study.
\end{abstract}

\footnotetext{
* Centro de Linguística da Universidade NOVA de Lisboa, Portugal.
}

\section{LINHA DÁGUA}


Then, we propose an analysis of the concepts underlying designations such as 'documentary language', 'controlled vocabulary' and 'knowledge organization system' in specialized texts. We conclude with an overview of SKOS (Simple Knowledge Organization System), a model to represent knowledge organization systems in the semantic web, which is evaluated in terms of its ability to model terminological resources according to the double-dimension approach and the main elements of the ISO 1087 standard.

Keywords: Knowledge Organization Systems; Controlled Vocabularies; Documentary Languages; Terminological Resources; SKOS.

\section{Introdução}

A linguística e a ciência da informação são duas áreas de conhecimento distintas, ainda que estejam relacionadas a diversos níveis, sobretudo no atual panorama académico marcado pela trans- e interdisciplinaridade. Uma das relações de maior relevo entre estas áreas pode ser estabelecida através da terminologia, entendida aqui como área de estudos de natureza bidimensional, linguística e conceptual, assumindo um papel de disciplina de interfaces entre as ciências da linguagem e as diversas áreas de conhecimento, em particular no que diz respeito ao léxico e às línguas de especialidade (COSTA; SILVA; BATISTA, 2020).

Deste ponto de vista, a terminologia relaciona-se com a organização do conhecimento (OC), um subdomínio da ciência da informação. A organização do conhecimento, ou para usar uma designação mais recente, sistemas de organização do conhecimento (SOC) (HJØRLAND, 2008; HODGE, 2000; ZENG, 2008), consiste na prática e estudo de atividades como a indexação por assuntos e classificação documental, ou a construção e aplicação das chamadas linguagens documentais e vocabulários controlados. Estes recursos incluem as classificações e listas de cabeçalhos de assuntos, muito utilizadas em bibliotecas, arquivos e outros serviços de informação, embora atualmente incluam tipos de recursos surgidos em ambiente digital, como as ontologias da representação do conhecimento e da web semântica (GUARINO; OBERLE; STAAB, 2009). A adoção das tecnologias de informação e o desenvolvimento da web levaram a transformações significativas nas tradicionais linguagens documentais, deixando estas de estar limitadas ao suporte em papel. A transição para o meio digital tornou viável, entre outros aspetos, o desenvolvimento de recursos de grandes dimensões e complexidade, abrangendo dezenas de milhares de conceitos com designações em diversas línguas, assim como a sua aplicação no enriquecimento e descoberta de dados em bibliotecas digitais e portais semânticos.

Neste artigo, assumimos que os SOC são recursos terminológicos e, como tal, podem ser abordados tendo em conta a bidimensionalidade da terminologia enquanto área de estudos das ciências da linguagem. Propomos, em seguida, uma análise dos conceitos acima referidos, desde linguagens documentais a vocabulários controlados e SOC, terminando com uma análise do SKOS (Simple Knowledge Organization System), um modelo para representação de SOC na web.

\section{LINHA DÁGUA}




\section{Terminologia e organização do conhecimento}

\subsection{A terminologia enquanto área de conhecimento}

O lexema terminologia, assim como os seus equivalentes noutras línguas, apresenta diversos significados, que abrangem o vocabulário utilizado em áreas especializadas, assim como a disciplina dedicada à recolha e estudo desse vocabulário. Neste sentido, Sager (1990) classificou o equivalente inglês, terminology, como termo polissémico impróprio (polysemous misnomer), uma vez que nenhum dos seus significados corresponde precisamente ao seu significado etimológico:

By its etymology 'terminology' would mean 'the science/study/knowledge of terms' which would make it parallel to lexicology, the science/study/knowledge of the lexicon or lexical items; this interpretation is, however, rejected by most terminologists. (SAGER, 1990, p. 3)

De facto, apesar da pluralidade de perspetivas sobre a terminologia enquanto área de conhecimento (CABRÉ, 2003; COSTA, 2006; FABER, 2012; FELBER, 1984; ISO 1087, 2019; ROCHE, 2007; TEMMERMAN, 2000; WÜSTER, 1979), verificamos que as diversas abordagens teóricas não identificam a terminologia com o estudo do léxico das línguas. Pelo contrário, estas abordagens reconhecem os seguintes aspetos:

- A terminologia envolve contributos teóricos e metodológicos de diversos domínios, tais como a linguística, a filosofia, a psicologia, a informática, entre outros.

- A terminologia é caracterizada pela complexidade das unidades terminológicas, as quais podem ser abordadas segundo múltiplos pontos de vista e a partir de diversas disciplinas.

Apesar dos diversos pontos em comum, não podemos deixar de salientar as diferenças mais significativas entre as diversas abordagens à terminologia:

- O posicionamento da terminologia, quer como ciência mais ou menos autónoma (ISO 1087, 2019; ROCHE, 2007; WÜSTER, 1979), quer como área de estudos interdisciplinar (CABRÉ, 2009; FABER, 2012; SAGER, 1990; TEMMERMAN, 2000).

- O foco na normalização de termos e conceitos (FELBER, 1984; WÜSTER, 1979) ou na descrição do uso dos termos em textos de especialidade (CABRÉ, 2009; FABER, 2012; SAGER, 1990; TEMMERMAN, 2000).

- $\quad$ Enfase nos termos (CABRÉ, 2009; SAGER, 1990; TEMMERMAN, 2000) ou nos conceitos (ISO 1087, 2019; ROCHE, 2007; WÜSTER, 1979), enquanto ponto de partida do trabalho terminológico e/ou elemento teórico central. 
- Conceção semiótica dos termos enquanto designações atribuídas convencionalmente a conceitos previamente definidos (ISO 1087, 2019; ROCHE, 2007; WÜSTER, 1979), ou conceção linguístico-discursiva dos termos, determinada pelo seu uso em textos de especialidade (CABRÉ, 2009; FABER, 2012; SAGER, 1990; TEMMERMAN, 2000).

Tendo em conta a natureza interdisciplinar deste domínio, devemos também salientar a diversidade de métodos utilizados na investigação e no trabalho terminológicos, que vão desde a linguística de corpus (BOWKER; PEARSON, 2002; MELBY, 2012) ao trabalho colaborativo e validação de dados terminológicos envolvendo especialistas de domínio (SILVA, 2014).

A diversidade de abordagens teóricas em terminologia manifesta-se de forma mais acentuada no que diz respeito aos aspetos que incluímos na dimensão conceptual, tais como conceitos, sistemas conceptuais e modelos para representação de conhecimento. A primeira abordagem teórica em terminologia, a teoria geral da terminologia (FELBER, 1984; WÜSTER, 1979), colocou a ênfase na dimensão conceptual como traço distintivo da terminologia em relação à linguística estruturalista. Wüster (1979) e Felber (1984) salientaram os contributos de áreas como a lógica, a ontologia e a ciência da informação na construção de sistemas conceptuais para o trabalho terminológico. Esta abordagem tem como descendentes diretos, atualmente, o Comité Técnico 37 da Organização Internacional de Normalização (ISO), dedicado à redação de normas internacionais em terminologia (ISO 704, 2009, p. 70; ISO 1087, 2019), e a ontoterminologia, que coloca a ênfase no desenvolvimento de recursos terminológicos baseados em ontologias para a representação do conhecimento (ROCHE, 2007).

Desde a década de 1990, várias abordagens teóricas procuraram posicionar a terminologia como área de estudos da linguística, embora reconhecendo a sua natureza interdisciplinar. É este o caso da teoria comunicativa da terminologia (CABRÉ, 2000, 2009), que elege o termo como elemento teórico central, concebido como unidade linguística, cognitiva e sociocomunicativa. $\mathrm{O}$ termo é abordado como uma unidade lexical que adquire um valor especializado, ou terminológico, no léxico dos especialistas de domínio. O conceito, elemento distintivo da terminologia wüsteriana, passa a ser entendido como um aspeto da unidade terminológica, podendo ser abordado como objeto de estudo da semântica lexical.

A teoria comunicativa da terminologia abriu caminho para abordagens baseadas em enquadramentos teóricos da semântica cognitiva, nomeadamente a terminologia sociocognitiva (TEMMERMAN, 2000) e a terminologia de marcos semânticos (frame-based terminology) (FABER, 2012). No caso da terminologia sociocognitiva, é dado destaque à metáfora conceptual e à semântica prototípica (LAKOFF, 1987; LAKOFF; JOHNSON, 1980) na descrição de unidades lexicais especializadas, enquanto que na terminologia de marcos semânticos o significado dos termos é descrito através de padrões de conhecimento baseados em marcos semânticos (FILLMORE, 1985).

\section{LINHA DÁGUA}




\subsubsection{A abordagem bidimensional à terminologia}

A abordagem bidimensional tem procurado afirmar-se nas últimas décadas como enquadramento teórico e metodológico baseado na distinção entre as dimensões linguística e conceptual da terminologia (COSTA, 2006, 2013; COSTA; SILVA; BATISTA, 2020; SANTOS; COSTA, 2015). Assim, partimos do pressuposto de que a terminologia é uma área de estudos interdisciplinar e que, como tal, nasce da confluência entre diversas áreas de conhecimento, incluindo vários subdomínios da linguística, filosofia e informática.

A distinção entre as dimensões linguística e conceptual no plano teórico facilita a conciliação de métodos semasiológicos, centrados na constituição e análise de corpora de textos de especialidade, e métodos onomasiológicos, centrados na elaboração de sistemas conceptuais. Enquanto a extração e o estudo de termos, colocações e marcadores linguísticos relevam da dimensão linguística, a modelização do conhecimento releva da dimensão conceptual, que é - por definição - extralinguística. O texto de especialidade é o elemento central do trabalho terminológico, já que ambas as metodologias requerem a certo ponto o recurso aos textos produzidos por especialistas e disseminados através das suas comunidades de prática, seja no meio académico, técnico ou artístico.

Em suma, a abordagem bidimensional baseia-se nos seguintes pressupostos:

- Reconhecimento da natureza interdisciplinar da terminologia enquanto área de estudos.

- Distinção entre uma dimensão linguística e uma dimensão conceptual na terminologia e no trabalho terminológico.

- Confluência de métodos semasiológicos e onomasiológicos.

- Papel central do texto de especialidade.

No contexto da investigação em semântica, o postulado de um nível de análise linguístico e um nível de análise conceptual não é novo. Encontramos eco deste postulado, por exemplo, em Wierzbicka (1996), quando defende a distinção entre o significado lexical e o conhecimento científico. Para esta autora, a distinção justifica-se pelo facto de as línguas e o conhecimento constituírem dois planos de análise distintos:

Science is, or tries to be, universal and to reflect the knowledge accumulated by mankind as a whole (and, more specifically, by the professional experts in different fields of knowledge); languages are not universal, and each of them reflects the experience of a particular part of mankind, united by a common culture and a common existential framework (WIERZBICKA, 1996, p. 338)

Seguindo o mesmo postulado, não identificamos a análise do significado de unidades lexicais especializadas com a organização do conhecimento, uma vez que esta última não releva de nenhum sistema linguístico. Este postulado facilita o trabalho de natureza trans- e interdisciplinar em terminologia, incluindo a constituição de corpora de especialidade e a

\section{LINHA DÁGUA}


extração semiautomática de termos (ALMEIDA; COSTA; ROCHE, 2019), a relação entre a terminologia e as ontologias da representação do conhecimento (ALMEIDA; COSTA, 2021) e a construção de vocabulários controlados e sua publicação na web como dados abertos e ligados (ALMEIDA; FREIRE; MONTEIRO, 2021).

Neste capítulo, descrevemos sumariamente a terminologia enquanto área de conhecimento e posicionámo-nos no contexto das diversas abordagens teóricas e metodológicas que têm vindo a ser propostas nas últimas décadas. Apresentaremos, em seguida, a OC como subdomínio da ciência da informação, tendo em conta ainda a sua relação com a terminologia.

\subsection{Organização do conhecimento (OC)}

De acordo com Hjørland (2008), a OC pode ser definida através dos seguintes objetos de estudo:

- Processos de organização do conhecimento, tais como a indexação de documentos por assuntos, isto é, a atribuição de descritores, ou termos de indexação, aos documentos e a classificação de documentos por temática ou área de conhecimento. Estes processos são tradicionalmente levados a cabo em bibliotecas, arquivos, museus e outras instituições de memória.

- Sistemas de organização do conhecimento utilizados nos processos acima referidos para organizar os documentos, as suas representações, as obras que os abrangem e os próprios conceitos das diversas áreas de conhecimento.

Este autor salienta, todavia, que não é possível fazer uma reflexão teórica rigorosa neste domínio isoladamente. Pelo contrário, a OC deverá ser abordada num sentido mais amplo, abrangendo o estudo da produção e disseminação do conhecimento nas sociedades. Hjørland (2008) nota que diversos domínios contribuem para esta abordagem interdisciplinar à OC, como por exemplo, a linguística, a psicologia e a sociologia. Apesar das diferentes metodologias e perspetivas disciplinares que separam estas áreas, Hjørland (2008) enfatiza a existência de um plano epistemológico comum, por exemplo, ao nível das teorias do conceito, as quais podem sustentar a investigação nas diversas disciplinas.

\subsubsection{A teoria do conceito em OC}

É na teoria do conceito que encontramos a relação mais direta entre terminologia e OC, sobretudo através do trabalho de Dahlberg $(1978,1992,2009)$. Esta autora propôs a chamada teoria analitica do conceito (referent-oriented, analytical concept theory), a qual ainda hoje transparece nas normas ISO sobre o vocabulário, princípios e métodos em terminologia (ISO 704, 2009; ISO 1087, 2019). Como refere Campos (2001), a teoria analítica do conceito foi inicialmente proposta como base para a terminologia das ciências sociais, em reação ao peso

\section{LINHA D'ÁGUA}


excessivo da engenharia e da normalização no trabalho de Wüster (1979). Em seguida, a teoria proposta por Dahlberg (1992) assumiu um papel central nos estudos sobre classificação, estendendo-se mais tarde à $\mathrm{OC}$.

Segundo Dahlberg (1992), o conhecimento é um estado de consciência em relação ao mundo, o qual se manifesta através da linguagem, mais especificamente, através de proposições verdadeiras sobre os referentes no mundo. A natureza analítica desta teoria deve-se à noção de que o conhecimento pode ser decomposto em conceitos, os quais correspondem a combinações únicas de características ou elementos conceptuais:

One cannot predicate a true fact without expressing one's knowledge of something. Thus every predication yields a knowledge element and the necessary sum total of predications can be synthesized into the corresponding knowledge unit. Concepts are thus the units of our knowledge, and this is an essential finding on which we can base all our activities in the area of knowledge organization and terminology. (DAHLBERG, 1992, p. 66)

Tanto a terminologia como a OC preconizam a criação de sistemas conceptuais de forma a tornar explícito o conhecimento do domínio de análise e a apoiar a redação de definições. Os sistemas conceptuais consistem em diversos tipos de relações entre conceitos, hierárquicas e não hierárquicas, tais como genéricas, partitivas e diversos tipos de relações associativas (NUOPPONEN, 2014).

Por outro lado, os termos correspondem a designações ou denominações de conceitos. Como tais, Dahlberg (1992) considera que os termos devem refletir as características essenciais dos respetivos conceitos através dos seus constituintes morfológicos ou lexicais. Por outro lado, os termos devem facilitar a comunicação especializada, permitindo formar paradigmas derivacionais. A internacionalização dos termos é também encorajada, por exemplo, através de empréstimos linguísticos de termos utilizados internacionalmente.

Embora a teoria analítica do conceito seja útil para a elaboração de recursos terminológicos, devemos salientar a existência de outras abordagens ao conceito na investigação em OC. Neste particular, Hjørland (2009) identificou quatro pontos de vista epistemológicos:

- Empirismo. Os conceitos devem ser definidos através da aglomeração de objetos com propriedades em comum.

- Racionalismo. Os conceitos devem ser definidos através da combinação de primitivos semânticos, tais como facetas ou características conceptuais indecomponíveis.

- Historicismo. Os conceitos devem ser definidos pela sua genealogia em relação às teorias e discursos que os sustentam.

- Pragmatismo. Os conceitos devem ser definidos pela sua utilidade num sistema e fixados através de signos.

\section{LINHA DÁGUA}


Como salienta Hjørland (2009), a investigação em sistemas de organização do conhecimento (SOC) exige o reconhecimento dos pontos de vista epistemológicos que subjazem a estes sistemas, uma vez que o conhecimento é sempre baseado em pressupostos:

Scientific observations, theories and concepts are always mediated by presumptions, and competing views and concepts exist in almost every field of knowledge. The most important task of research on KOS [knowledge organization systems] is to argue which conceptions should be preferred as the basis on which to construe and evaluate KOS. (HJØRLAND, 2008, p. 1528-1529)

No capítulo seguinte, abordaremos os recursos terminológicos produzidos e estudados pela OC, os quais têm sido designados por diversos termos, tais como linguagens documentais, vocabulários controlados e SOC.

\section{Recursos terminológicos em OC: linguagens, vocabulários e sistemas}

$\mathrm{Na}$ literatura em ciência da informação, verifica-se uma proliferação de termos genéricos para designar os recursos tradicionalmente utilizados em bibliotecas e outras unidades de informação para a $\mathrm{OC}$, desde linguagem documental a sistema de organização do conhecimento. Podemos agrupar estas designações da seguinte forma:

- $\quad$ linguagem documental, documentária ou de indexação;

- vocabulário controlado ou estruturado,

- $\quad$ sistema de organização do conhecimento.

Propomos, na próxima secção, uma breve análise dos conceitos subjacentes a estas designações em OC.

\subsection{Linguagens documentais, documentárias ou de indexação}

O primeiro conjunto de termos listado acima inclui as designações mais antigas nos textos de especialidade. O uso dos equivalentes em inglês (documentary language) e francês (langage documentaire) é atestado nas normas internacionais em informação e documentação, pelo menos desde a década de $1980^{1}$. Linguagem possui aqui o mesmo significado relativamente aos termos complexos linguagem artificial ou linguagem de programação, ou seja, é entendida como sistema formal e explícito de signos.

1 De acordo com a informação recolhida, a redação da antiga norma ISO 5127-6:1983 - Documentation and information - vocabulary - Part 6: Documentary languages, terá começado em 1979. Disponível em: https://www.iso.org/ Acesso em: 06 jul. 2021.

\section{LINHA DÁGUA}


Para Campos (2001, p. 17), as linguagens documentais são definidas através da sua função como "instrumentos utilizados para representar o conhecimento de uma dada área do saber". Esta autora observa a base formal e conceptual destas linguagens, nas quais os conceitos são identificados por símbolos, de forma a permitir a sua manipulação:

Os conceitos, para serem manipulados, necessitam de um símbolo que permita a comunicação. Na área da documentação, o símbolo é lingüístico, sendo denominado "termo de recuperação". Os conceitos e termos são, portanto, elementos de qualquer esquema de classificação e dos tesauros. (CAMPOS, 2001, p. 17)

Esta formulação também está patente nas normas portuguesas e internacionais, em que linguagem documental é definida como "linguagem formal utilizada para caracterizar os dados ou o conteúdo de documentos e permitir o seu armazenamento e recuperação" (NP 4285-4, 2000, §4.1.1-01). Na mesma linha, o termo indexing language é definido como "artificial language established to characterize the content or form of a document" (ISO 5127, 2017, §3.8.1.06). Ao contrário de uma língua natural, uma linguagem formal ou artificial caracterizase por ter regras explícitas, conhecidas a priori, no que diz respeito ao seu léxico (ou vocabulário), sintaxe e semântica.

No caso das linguagens documentais com base nas línguas naturais, tais como os tesauros e as listas de cabeçalhos, o controlo ou normalização do vocabulário é um elementochave. O manual SIPORbase (Sistema de Indexação em Português), muito utilizado nas bibliotecas portuguesas, consiste precisamente num conjunto de regras para a normalização dos termos: "O vocabulário documental é controlado, face à linguagem natural, em dois aspectos essenciais: a forma dos termos e o seu significado." (ÁREA DE CLASSIFICAÇÃO E INDEXAÇÃO DA BIBLIOTECA NACIONAL, 1998, f. 3, p. 4). Neste particular, o controlo do vocabulário inclui a limitação do número de conceitos e termos, a desambiguação entre homónimos (através da adição de qualificadores parentéticos) ${ }^{2}$, a limitação do âmbito de aplicação dos termos (através de notas de âmbito) ${ }^{3}$ e o controlo da sinonímia (distinguindo entre descritores e não-descritores).

\subsection{Vocabulários controlados e vocabulários estruturados}

De acordo com Harpring (2010), os vocabulários controlados são ferramentas que promovem a consistência da indexação de documentos em catálogos documentais, através da distinção entre termos preferenciais e variantes. Por outro lado, os vocabulários controlados

2 Os qualificadores parentéticos são adicionados aos termos para fins de desambiguação (ISO 25964-1, 2011, $\S 6.2 .2$ ). Por exemplo, podemos querer distinguir entre os conceitos de teatro enquanto instituição e teatro enquanto edifício: "teatros (instituições)" e "teatros (edifícios)".

3 As notas de âmbito têm como função clarificar o uso de um conceito de um vocabulário controlado no âmbito da indexação por assuntos (ISO 25964-1, 2011, §5.2). Por exemplo, o conceito de "iluminuras" num vocabulário controlado poderá incluir a seguinte nota de âmbito: “Abrange tanto a decoração ornamental como as ilustrações em manuscritos e incunábulos, desde que feitas à mão”.

\section{LINHA DÁGUA}


facilitam a recuperação da informação, pois levam os utilizadores dos catálogos a utilizar os mesmos termos atribuídos pelos indexadores:

A controlled vocabulary is an organized arrangement of words and phrases used to index content and/or to retrieve content through browsing or searching. It typically includes preferred and variant terms and has a defined scope or describes a specific domain. (HARPRING, 2010, p. 12)

Como a autora refere, os vocabulários controlados são muitas vezes também vocabulários estruturados, no sentido em que dão ênfase às relações entre os conceitos denotados pelos termos. Neste sentido, as atuais normas ISO em informação e documentação tornam mais explícita a dimensão conceptual dos vocabulários controlados. As normas especificam também que um vocabulário controlado não contém necessariamente termos das línguas naturais, podendo consistir em códigos alfanuméricos ou outro tipo de símbolos, com regras próprias para a sua elaboração. Assim, controlled vocabulary é definido nas normas como "prescribed list of terms, headings or codes, each representing a concept" (ISO 25964-1, $2011, \S 2.12$ ), enquanto que structured vocabulary é entendido como "organized set of terms, headings or codes representing concepts and their inter-relationships, which can be used to support information retrieval" (ISO 25964-1, 2011, §2.56).

\subsection{Sistemas de organização do conhecimento (SOC)}

A designação sistema de organização do conhecimento, e seus equivalentes, é mais recente nos textos de especialidade. No entanto, o conceito de SOC não surge nas normas internacionais em informação e documentação, tais como a ISO 5127 (2017) e a ISO 25964-1 (2011), nas quais os equivalentes ingleses de linguagem de indexação e vocabulário controlado são utilizados. O conceito de SOC é, apesar disso, predominante na literatura científica em OC, uma vez que engloba não só as linguagens documentais utilizadas em bibliotecas e outras unidades de informação, mas também os modelos formais para representação do conhecimento na web, como é o caso das ontologias.

Hodge (2000) avançou com uma das primeiras tentativas de definição do conceito de SOC, abrangendo as linguagens documentais, vocabulários controlados e os esquemas de conceitos da web semântica:

The term knowledge organization systems is intended to encompass all types of schemes for organizing information and promoting knowledge management. Knowledge organization systems include classification schemes that organize materials at a general level (such as books on a shelf), subject headings that provide more detailed access, and authority files that control variant versions of key information (such as geographic names and personal names). They also include less-traditional schemes, such as semantic networks and ontologies. (HODGE, 2000, p. 3)

Moreiro González (2011) salienta que todos os recursos designados por linguagens documentais são SOC, embora variem em termos de estrutura e composição, desde listas de

\section{LINHA DÁGUA}


palavras-chave até recursos baseados em conceitos e relações conceptuais, tais como os tesauros e as ontologias. Por outro lado, como salienta o mesmo autor, nem todos os SOC podem ser caracterizados como vocabulários controlados, apesar da ênfase das tradicionais linguagens documentais no controlo terminológico. As chamadas folksonomias, isto é, conjuntos de palavras-chave livremente atribuídas pelos utilizadores de um catálogo, portal ou outro tipo de sítio web, são exemplos de SOC em que não se verifica controlo de vocabulário (MOREIRO GONZÁLEZ, 2011).

Como podemos verificar, o conceito de SOC permanece algo difuso, abrangendo diversos tipos de recursos terminológicos. Veremos, em seguida, algumas das suas tipologias mais relevantes na literatura de especialidade.

\subsection{Tipologias de SOC}

A tabela 1 apresenta a tipologia de SOC proposta por Hodge (2000), a qual serviu de base para as propostas subsequentes de diversos autores. Hodge agrupa os SOC em três categorias empíricas: (i) listas de termos, (ii) classificações e categorias e (iii) listas de relações.

Tabela 1: Tipologia de SOC de acordo com Hodge (2000)

\begin{tabular}{|l|l|}
\hline \multirow{4}{*}{ Categorias } & Tipos de SOC \\
\hline \multirow{4}{*}{ Listas de termos } & Ficheiros de autoridade \\
\cline { 2 - 2 } & Índices toponímicos \\
\cline { 2 - 2 } & Glossários \\
\cline { 2 - 2 } & Dicionários \\
\hline \multirow{4}{*}{ Listassificações e categorias } & Listas de cabeçalhos de assunto \\
\cline { 2 - 2 } & Esquemas de classificação \\
\cline { 2 - 2 } & Taxonomias \\
\hline & Tesauros \\
\cline { 2 - 2 } & Redes semânticas \\
\cline { 2 - 2 } & Ontologias \\
\hline
\end{tabular}

Fonte: elaborada pelo autor.

Segundo esta proposta, as listas de termos incluem os ficheiros de autoridade, ou seja, listas para controlo das designações autorizadas e variantes para pessoas, organizações, nomes geográficos e outro tipo de entidades. Hodge (2000) salienta, todavia, que os ficheiros de autoridade podem estar organizados de acordo com um esquema de classificação e podem mesmo conter alguma estrutura hierárquica. A autora acrescenta ainda, nas listas de termos, os índices toponímicos (gazetteers, em inglês), em que cada entrada poderá incluir o tipo de lugar

\section{LINHA DÁGUA}


(p. ex., país, rio, edifício) e coordenadas geográficas. Finalmente, Hodge (2000) integra nesta categoria os glossários e dicionários, embora não clarifique a utilização destes recursos lexicográficos na organização e recuperação de informação.

As classificações e categorias incluem, de acordo com Hodge (2000), as listas de cabeçalhos de assunto, que consistem em listas de descritores para os assuntos dos documentos numa coleção, juntamente com regras para a sua combinação. Desta categoria, fazem ainda parte os esquemas de classificação, os quais permitem reunir documentos nas mesmas classes e subclasses, e as taxonomias, que consistem numa estrutura hierárquica de classes, sendo muito usadas em empresas no contexto de sistemas de gestão do conhecimento.

Hodge (2000) introduz nas chamadas listas de relações os tesauros, responsáveis pela organização dos conceitos num domínio, através de relações hierárquicas, associativas e instanciais, apresentando os termos preferenciais e não preferenciais para cada conceito. As redes semânticas, por seu turno, tendem a dar mais ênfase às relações não hierárquicas entre conceitos, incluindo diversos tipos destas relações (por exemplo, causa-efeito, processoagente). Por último, as ontologias constituem o tipo de SOC mais recente, sendo definidas como modelos de conhecimento num qualquer domínio. Enquanto modelos formais, as ontologias baseiam-se em axiomas, ou declarações formais, para descrição das classes, propriedades e indivíduos num domínio do conhecimento.

Outra tipologia influente foi proposta por Zeng (2008). Esta tipologia é baseada na estrutura e função dos SOC, consistindo nas seguintes categorias: (i) listas de termos, (ii) modelos semelhantes a metadados, (iii) classificações e categorizações e (iv) modelos relacionais.

Na sua generalidade, as listas de termos e os modelos semelhantes a metadados são planos, embora os índices toponímicos, diretórios e ficheiros de autoridade possam ter alguma estrutura. As classificações e categorizações apresentam uma estrutura bidimensional, através de relações hierárquicas entre as classes, categorias ou assuntos. Finalmente, os modelos relacionais caracterizam-se pela sua complexidade, podendo apresentar diversos tipos de relações não hierárquicas.

Zeng (2008) baseia a sua tipologia nas seguintes funções: (i) eliminar a ambiguidade, (ii) controlo dos sinónimos, (iii) estabelecer relações hierárquicas entre conceitos, (iv) estabelecer relações associativas entre conceitos, e (v) explicitar as propriedades ou atributos.

Como testemunho da natureza difusa do conceito, Souza, Tudhope e Almeida (2012) incluem ainda como categorias de SOC o texto não estruturado (por exemplo, os resumos), as linhas de concordâncias da linguística de corpus e os modelos e diagramas conceptuais. Esta perspetiva, no entanto, vai além da relação com a terminologia, inserindo-se no sentido mais lato da OC enquanto área de estudos (HJØRLAND, 2008).

\section{LINHA DÁGUA}


Tabela 2: Tipologia de SOC de acordo com Zeng (2008)

\begin{tabular}{|c|c|}
\hline Categorias & Tipos de SOC \\
\hline \multirow{4}{*}{ Listas de termos } & Listas de palavras-chave \\
\hline & Dicionários \\
\hline & Glossários \\
\hline & Anéis de sinónimos \\
\hline \multirow{3}{*}{$\begin{array}{l}\text { Modelos semelhantes a } \\
\text { metadados }\end{array}$} & Ficheiros de autoridade \\
\hline & Diretórios \\
\hline & Índices toponímicos \\
\hline \multirow{4}{*}{ Classificações e categorizações } & Listas de cabeçalhos de assunto \\
\hline & Esquemas de categorização \\
\hline & Taxonomias \\
\hline & Esquemas de classificação \\
\hline \multirow{3}{*}{ Modelos relacionais } & Tesauros \\
\hline & Redes semânticas \\
\hline & Ontologias \\
\hline
\end{tabular}

Fonte: elaborada pelo autor.

Como referido por Bratková e Kučerová (2014), a proposta de Zeng (2008) que descrevemos acima foi posteriormente revista e transformada no KOS Types Vocabulary ${ }^{4}$, que fornece os tipos de SOC a serem indicados no NKOS (Networked Knowledge Organization Systems $)^{5}$, um perfil de metadados para descrição de SOC na web. Uma das alterações mais significativas consistiu na adição das terminologias, entendidas como conjuntos de conceitos e designações em domínios de especialidade ou assuntos (ISO 1087, 2019, §3.1.11).

Os principais tipos de SOC listados no KOS Types Vocabulary serviram também como base para a norma ISO relativa à interoperabilidade dos tesauros com outros tipos de SOC (designados aqui por vocabulários), incluindo apenas os esquemas de classificação, as taxonomias, os cabeçalhos de assunto, as ontologias, as terminologias, os ficheiros de autoridades de nomes e os anéis de sinónimos (ISO 25964-2, 2013). Esta tipologia mais restrita integra apenas os SOC normalmente utilizados para a recuperação de informação, ou que possam ser reutilizados para a construção de tesauros. Por exemplo, as terminologias podem constituir-se como fontes de vocabulário para a construção de tesauros com vista à recuperação de informação.

4 Disponível em https://nkos.slis.kent.edu/nkos-type.html Acesso em: 06 jul. 2021.

5 Disponível em: https://nkos.slis.kent.edu/nkos-ap.html Acesso em: 06 jul. 2021.

\section{LINHA DÁGUA}




\section{SKOS (Simple Knowledge Organization System): modelização de SOC na web}

O SKOS (Simple Knowledge Organization System) é uma recomendação do W3C (World Wide Web Consortium) para a modelização de SOC através de tecnologias da web semântica ${ }^{6}$. Desenvolvido no seguimento de vários projetos europeus desde o final da década de 1990, o SKOS foi concebido para a migração de SOC para a web, nomeadamente através da sua representação em RDF (Resource Description Framework) ${ }^{7}$, um dos fundamentos da web semântica (HITZLER; KRÖTZSCH; RUDOLPH, 2010).

A motivação para o desenvolvimento do SKOS é elucidativa do fosso entre as tradicionais linguagens documentais e os modelos formais para representação do conhecimento. Como referem Baker et al. (2013), instrumentos como os tesauros e os esquemas de classificação constituem SOC informais, ao contrário das ontologias. Como tal, é inviável a tradução direta destes sistemas para axiomas de classes e propriedades:

Informally defined KOSs cannot typically be translated into the language of RDFS and OWL properties and classes, with their formal-logical implications, without introducing potentially false or misleading logical precision. Informal KOSs may be converted into formal ontologies [...], but the process of assigning appropriate formal semantics to the elements of a KOS may require a long, hard modeling effort. (BAKER et al., 2013, p. 38)

Os autores avançam com o exemplo das relações hierárquicas, que num tesauro, abrangem tipicamente as relações genéricas, partitivas e de instanciação (ISO 25964-1, 2011). A tradução destas relações para uma ontologia requer a desambiguação entre relações de subsunção (classe-subclasse), de classe-indivíduo, parte-todo e possivelmente outras, dada a natureza imprecisa destes recursos. Por outro lado, os tradicionais vocabulários controlados mantêm-se relevantes hoje, com aplicações que vão desde a melhoria da precisão na recuperação de informação à expansão da pesquisa. Desta forma, o SKOS permite uma transição de baixo custo de SOC informais para a web semântica (BAKER et al., 2013).

O modelo de dados do SKOS (Fig. 1) permite representar os SOC como esquemas de conceitos. O SKOS define conceito enquanto unidade de pensamento, ecoando as definições presentes em versões mais antigas das normas ISO em terminologia. No entanto, esta definição é apenas sugestiva, uma vez que deve abranger a organização intelectual da generalidade dos SOC, embora se aplique mais diretamente aos tesauros e esquemas de classificação enquanto exemplos prototípicos da aplicação do SKOS.

Os conceitos podem se relacionar entre si através de relações semânticas, constituindo hierarquias informais (broader, narrower) ou redes de associações (related). Por outro lado, os

6 SKOS Simple Knowledge Organization System Reference. Disponível em: https:/www.w3.org/TR/skosreference/ Acesso em: 06 jul. 2021.

7 Resource Description Framework. Disponível em: https://www.w3.org/RDF/ Acesso em: 06 jul. 2021.

\section{LINHA DÁGUA}


conceitos podem ser incluídos em coleções não hierárquicas (simples ou ordenadas), sendo designados por formas lexicais (lexical labels) - preferenciais, alternativas e rejeitadas - e identificadas por notações alfanuméricas.

Existem também propriedades de documentação que permitem associar diversos tipos de notas, exemplos e definições. As propriedades de mapeamento permitem estabelecer relações com conceitos de esquemas externos, o que é essencial para a publicação de SOC como dados ligados (linked data $)^{8}$.

Figura 1: Modelo de dados do SKOS (baseado em Baker et al., 2013)

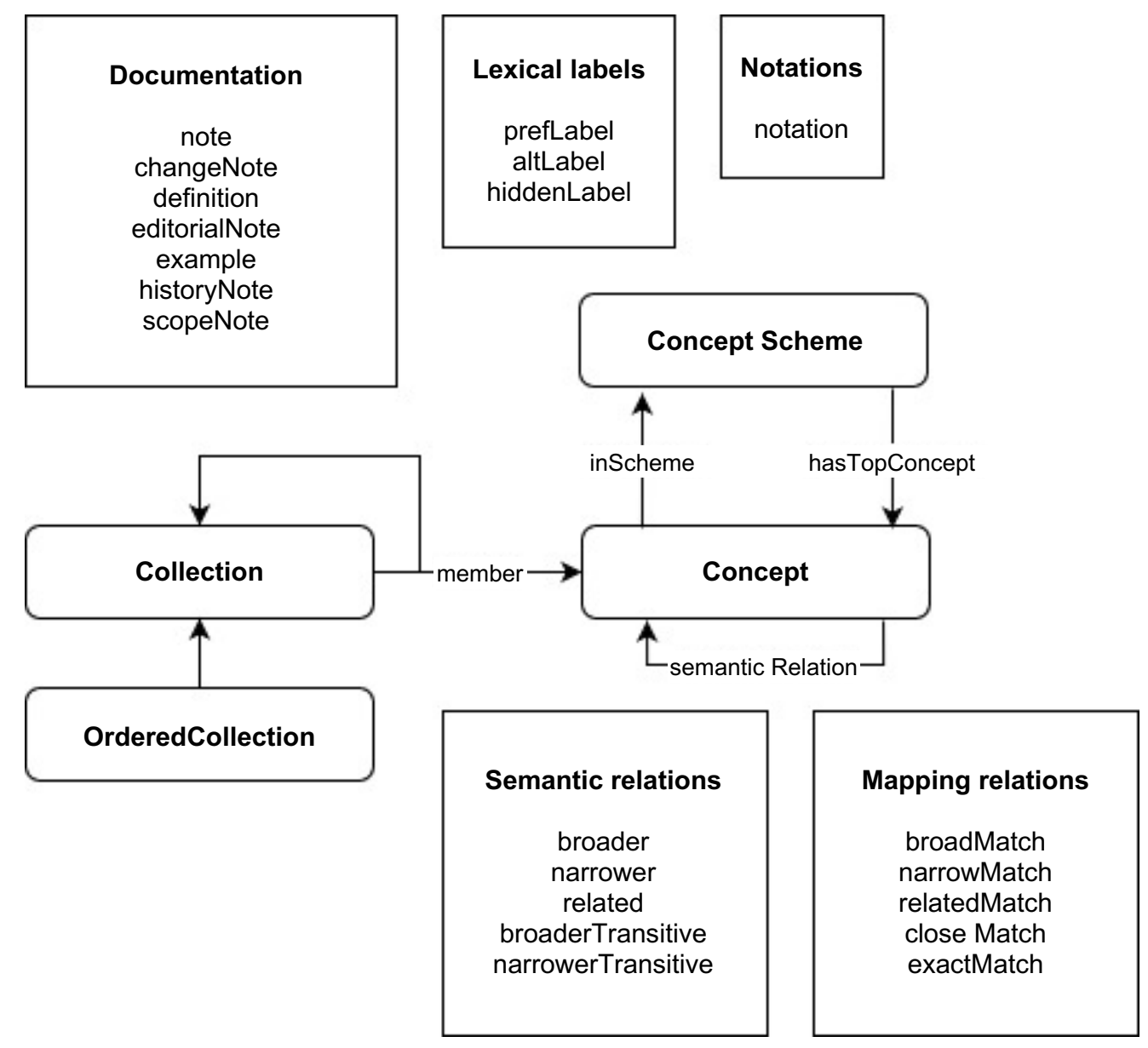

Fonte: elaborada pelo autor.

Uma das limitações do SKOS consiste na representação dos termos, uma vez que o SKOS apenas permite que os conceitos sejam designados por etiquetas lexicais, as quais correspondem linguisticamente a formas de termos simples, compostos ou complexos. Tal implica que as formas singular e plural do mesmo termo (por exemplo, gato e gatos) possam designar o mesmo conceito num esquema conceptual, enquanto etiquetas preferencial e

8 Linked Data. Disponível em: https://www.w3.org/wiki/LinkedData Acesso em: 06 jul. 2021.

\section{LINHA DÁGUA}


alternativa. Por outro lado, o SKOS não permite representar qualquer tipo de informação associada às formas lexicais, como é o caso das relações lexicais ou semânticas entre termos da mesma língua.

Para permitir uma maior flexibilidade na modelização das formas lexicais, foi introduzida a extensão SKOS-XL (SKOS eXtension for Labels $)^{9}$, que inclui a classe Label para as formas lexicais, assim como uma propriedade genérica para estabelecer relações ao nível das formas lexicais, podendo esta propriedade ser especializada através da definição de subpropriedades para representar relações lexicais específicas. Isto permite, por exemplo, representar a relação entre as formas Países Africanos de Lingua Oficial Portuguesa e o seu acrónimo $P A L O P$, o que não seria possível no modelo SKOS simples.

\subsection{O SKOS do ponto de vista da bidimensionalidade da terminologia}

A crescente aproximação entre as normas internacionais em terminologia e em informação e documentação traduz-se também ao nível dos modelos para representação de SOC, como é o caso do SKOS. Tendo em conta que as terminologias podem ser incluídas nesta categoria de recursos, ainda que com finalidades e características diferentes, importa avaliar a capacidade do SKOS em modelizar terminologias, entendidas como conjuntos de conceitos e termos em domínios ou assuntos especializados.

Tomando a norma ISO 1087 (2019) como referência, verificamos que um recurso terminológico é constituído por um conjunto de entradas terminológicas, as quais reúnem dados sobre um só conceito e as suas designações, sejam termos em línguas naturais, nomes próprios ou símbolos. A informação a apresentar sobre os conceitos numa entrada terminológica deverá incluir relações conceptuais (hierárquicas ou associativas), designações, tais como termos, símbolos ou nomes próprios (no caso dos chamados conceitos individuais) e definições. Poderá ainda integrar informação sobre os termos preferenciais, admitidos, rejeitados ou obsoletos para um mesmo conceito, contextos de uso e relações léxico-semânticas (sinonímia, equivalência e antonímia).

A tabela 3 apresenta correspondências possíveis entre os elementos da ISO 1087 acima indicados e o vocabulário do SKOS. Aí verificamos que o SKOS permite modelizar a grande maioria dos elementos presentes na norma sobre terminologia, embora alguns elementos (como as relações léxico-semânticas) exijam a definição de propriedades através da extensão SKOS$\mathrm{XL}^{10}$.

9 Appendix B. SKOS eXtension for Labels (SKOS-XL). Disponível em: https://www.w3.org/TR/skosreference/\#xl Acesso em: 06 jul. 2021.

10 Existe também uma limitação no que diz respeito à indicação de símbolos não linguísticos como designações. Embora seja possível fazê-lo através da propriedade notation do SKOS, dois conceitos não podem ter a mesma notação.

\section{LINHA DÁGUA}


Tabela 3: Correspondência entre elementos ISO 1087 e SKOS

\begin{tabular}{|l|l|}
\hline Elemento ISO $\mathbf{1 0 8 7}$ & Elemento SKOS \\
\hline Recurso terminológico & Concept scheme \\
\hline Conceito (geral ou individual) & Concept \\
\hline Relação hierárquica (genérica, partiviva) & Broader, narrower \\
\hline $\begin{array}{l}\text { Relação associativa (sequencial, espacial, } \\
\text { temporal, causal) }\end{array}$ & Related \\
\hline Critério de subdivisão & Collection \\
\hline Definição (por intensão, por extensão) & Definition \\
\hline Designação (termo, nome próprio, símbolo) & Lexical label, notation \\
\hline Termo preferencial & Preferred label \\
\hline Termo admitido & Alternative label \\
\hline Termo rejeitado & Hidden label \\
\hline Termo obsoleto & {$[$ Não existe] } \\
\hline Contexto & Example \\
\hline Sinonímia & {$[$ Através do SKOS-XL] } \\
\hline Equivalência & {$[$ Através do SKOS-XL] } \\
\hline Antonímia & {$[$ Através do SKOS-XL] } \\
\hline
\end{tabular}

Fonte: elaborada pelo autor.

Uma outra questão pertinente prende-se com o controlo do vocabulário. O SKOS permite o controlo do vocabulário através da indicação de uma forma preferencial para cada conceito, a qual não pode ser associada a mais nenhum conceito. No entanto, a atribuição de etiquetas preferenciais não é obrigatória no SKOS, o que possibilita a modelização de recursos terminológicos sem controlo de vocabulário. Neste caso, os conceitos seriam representados apenas por etiquetas alternativas (que podem ser atribuídas a mais do que um conceito).

Em suma, verificamos que o SKOS estabelece a distinção entre as dimensões linguística e conceptual do trabalho terminológico, sendo flexível o suficiente para a modelização de recursos terminológicos baseados no conhecimento. A extensão SKOS-XL permite ainda modelizar relações léxico-semânticas entre designações.

\section{Conclusão}

Neste artigo, partimos do pressuposto que as ferramentas utilizadas na organização do conhecimento, desde tesauros a esquemas de classificação, podem ser entendidas como recursos terminológicos. Começamos por abordar as relações teóricas e metodológicas entre a terminologia e a organização do conhecimento, áreas de saber marcadas pela interdisciplinaridade.

\section{LINHA DÁGUA}


Assumindo como enquadramento teórico uma abordagem bidimensional, baseada na distinção entre a dimensão linguística e a dimensão conceptual da terminologia, propomos uma breve análise dos conceitos subjacentes às designações linguagem documental, vocabulário controlado e sistema de organização do conhecimento. Verificamos que os conceitos subjacentes não são idênticos, embora as suas designações sejam por vezes utilizadas de forma indiscriminada: existem SOC onde não se verifica controlo de vocabulário (por exemplo, as chamadas folksonomias) e que não podem ser caracterizadas como linguagens documentais (por exemplo, as ontologias). Este desfasamento ao nível das designações é indicativo da grande disparidade de tipologias de SOC nos textos de especialidade e reflete uma conceção mais abrangente da OC enquanto disciplina.

A nossa análise termina com a descrição do SKOS (Simple Knowledge Organization System), um modelo para a representação de SOC na web semântica. Avaliamos o modelo segundo a abordagem bidimensional da terminologia, aferindo a sua capacidade para representar os principais elementos das entradas terminológicas. Segundo a nossa análise, o SKOS, incluindo a sua extensão para as etiquetas lexicais, permite modelizar a grande maioria dos elementos presentes na norma ISO 1087, em que se definem os fundamentos e vocabulário da terminologia enquanto disciplina.

Este artigo vem, desta forma, confirmar a crescente aproximação teórica e metodológica entre a terminologia e a OC. A aproximação entre as referidas áreas manifesta-se também nas normas internacionais em ciência da informação, em que foram transpostos conceitos basilares da terminologia. Por exemplo, na norma ISO 5127, as definições de objeto, conceito e característica, entre outras, têm como fonte a ISO 1087. A aproximação entre terminologia e OC leva a que as ferramentas da OC, desde as linguagens documentais aos mais recentes SOC da web semântica, possam ser abordadas como recursos terminológicos de pleno direito. As implicações desta abordagem são significativas para a terminologia, que pode tomar partido de modelos como o SKOS para a representação de recursos terminológicos na web semântica, de forma interoperável com os SOC. Por outro lado, esta abordagem tem vantagens para a própria OC, onde podem ser aplicados enquadramentos teóricos nascidos no seio da terminologia, como é o caso da abordagem bidimensional, para a construção e análise de SOC. Tal perspetiva implica o reconhecimento das dimensões linguística e conceptual da terminologia, abrindo a possibilidade de aplicar métodos semasiológicos, baseados na análise de corpora linguísticos, em paralelo a métodos onomasiológicos, focados na análise do conhecimento em domínios especializados.

\section{Referências}

ALMEIDA, B.; COSTA, R. OntoAndalus: an ontology of Islamic artefacts for terminological purposes. Semantic Web Journal, v. 12, n. 2, p. 295-311, 2021.

\section{LINHA DÁGUA}


ALMEIDA, B.; COSTA, R.; ROCHE, C. The names of lighting artefacts: extraction and representation of Portuguese and Spanish terms in the archaeology of al-Andalus. Revue TAL, v. 60, n. 3, p. 113-137, 2019.

ALMEIDA, B.; FREIRE, N.; MONTEIRO, D. The development of the ROSSIO Thesaurus: supporting content discovery and management in a research infrastructure. DOSSO, D.; FERILLI, S.; MANGHI, P.; POGGI, A.; SERRA, G.; SILVELLO, G. In: (Eds.). Proceedings of the 17th Italian Research Conference on Digital Libraries. Aachen: CEUR-WS, 2021. Disponível em: http://ceur-ws.org/Vol-2816/ Acesso em: 06 jul. 2021.

ÁREA DE CLASSIFICAÇÃO E INDEXAÇÃO DA BIBLIOTECA NACIONAL. SIPORbase: Sistema de Indexação em Português : manual. 3a ed. rev. e aumentada. Lisboa: Biblioteca Nacional, 1998.

BAKER, T.; BECHHOFER, S.; ISAAC, A.; MILES, A.; SCHREIBER, G.; SUMMERS, S. Key Choices in the Design of Simple Knowledge Organization System (SKOS). Journal of Web Semantics, v. 20, p. 35-49, maio 2013.

BOWKER, L.; PEARSON, J. Working with specialized language : a practical guide to using corpora. London: Routledge, 2002.

BRATKOVÁ, E.; KUČEROVÁ, H. Knowledge Organization Systems and Their Typology. Revue of Librarianship, v. 25, n. 2, p. 1-25, 2014.

CABRÉ, M. T. Terminologie et linguistique : la théorie des portes. Terminologies nouvelles, $\mathrm{n}$. 21, p. 10-15, 2000.

CABRÉ, M. T. Theories of terminology: their description, prescription and explanation. Terminology, v. 9, n. 2, p. 163-199, 2003.

CABRÉ, M. T. La teoría comunicativa de la terminología: una aproximación lingüística a los términos. Revue française de linguistique appliquée, v. 14, n. 2, p. 9-15, 2009.

CAMPOS, M. L. A. Linguagem documentária: teorias que fundamentam sua elaboração. Niterói: Universidade Federal Fluminense, 2001.

COSTA, R. Plurality of theoretical approaches to terminology. In: PICHT, H. (Ed.). Modern approaches to terminological theories and applications. Bern: Peter Lang, 2006. p. 79-89.

COSTA, R. Terminology and specialised lexicography: two complementary domains. Lexicographica, v. 29, n. 1, p. 29-42, 2013.

COSTA, R.; SILVA, R.; CAMPOS, M. I. B. Terminologia, uma disciplina de interfaces. Linha d'Água, v. 33, n. 1, p. 1-8, abr. 2020.

DAHLBERG, I. A referent-oriented, analytical concept theory for INTERCONCEPT. International Classification, v. 5, n. 3, p. 142-151, 1978.

DAHLBERG, I. Knowledge organization and terminology: philosophical and linguistic bases. International Classification, v. 19, n. 2, p. 65-71, 1992.

DAHLBERG, I. Brief communication: Concepts and terms - ISKO's major challenge. Knowledge Organization, v. 36, n. 2/3, p. 169-177, 2009.

FABER, P. (Ed.). A cognitive linguistics view of terminology and specialized language. Berlin: De Gruyter Mouton, 2012.

FELBER, H. Terminology manual. Paris: UNESCO, 1984.

\section{LINHA DÁGUA}


FILLMORE, C. J. Frames and the semantics of understanding. Quaderni di semantica, v. 6, n. 2, p. 222-254, dez. 1985.

GUARINO, N.; OBERLE, D.; STAAB, S. What is an ontology? In: STAAB, S.; STUDER, R. (Eds.). Handbook on ontologies. Second ed. Berlin: Springer, 2009. p. 1-17.

HARPRING, P. Introduction to controlled vocabularies: terminology for art, architecture, and other cultural works. Los Angeles: Getty Research Institute, 2010.

HITZLER, P.; KRÖTZSCH, M.; RUDOLPH, S. Foundations of semantic web technologies. Boca Raton: CRC Press, 2010.

HJØRLAND, B. What is Knowledge Organization (KO)? Knowledge Organization, v. 35, n. 2/3, p. 86-101, 2008.

HJØRLAND, B. Concept theory. Journal of the American Society for Information Science and Technology, v. 60, n. 8, p. 1519-1536, 2009.

HODGE, G. Systems of Knowledge Organization for Digital Libraries: Beyond Traditional Authority Files. [S.1.] Council on Library and Information Resources, 2000.

ISO 704. Terminology work - Principles and methods. Geneva: ISO, 2009.

ISO 1087. Terminology work and terminology science - Vocabulary. Geneva: ISO, 2019.

ISO 5127. Information and documentation - Foundation and vocabulary. Geneva: ISO, 2017.

ISO 25964-1. Information and documentation - Thesauri and interoperability with other vocabularies - Part 1: Thesauri for information retrieval. Geneva: ISO, 2011.

ISO 25964-2. Information and documentation - Thesauri and interoperability with other vocabularies - Part 2: Interoperability with other vocabularies. Geneva: ISO, 2013.

LAKOFF, G. Women, fire and dangerous things: what categories reveal about the mind. Chicago: University of Chicago Press, 1987.

LAKOFF, G.; JOHNSON, M. Metaphors we live by. Chicago: University of Chicago Press, 1980.

MELBY, A. K. Terminology in the age of multilingual corpora. The Journal of Specialised Translation, n. 18, p. 7-29, jul. 2012.

MOREIRO GONZÁLEZ, J. A. Linguagens documentárias e vocabulários semânticos para a web: elementos conceituais. Salvador: EDUFBA, 2011.

NP 4285-4. Documentação e informação - Vocabulário - Parte 4: Linguagens documentais. Caparica: IPQ, 2000.

NUOPPONEN, A. Tangled web of concept relations: concept relations for ISO 1087-1 and ISO 704. TKE 2014: Ontology, Terminology \& Text Mining, n. p., jun. 2014.

ROCHE, C. Terme et concept : fondements pour une ontoterminologie. TOTh 2007. Annecy: Institut Porphyre, 2007.

SAGER, J. C. A practical course in terminology processing. Amsterdam: John Benjamins, 1990.

SANTOS, C.; COSTA, R. Domain specificity: semasiological and onomasiological knowledge representation. In: KOCKAERT, H. J.; STEURS, F. (Eds.). Handbook of terminology. Amsterdam: John Benjamins, 2015. v. 1, p. 153-179.

\section{LINHA DÁGUA}


SILVA, R. Gestão de terminologia pela qualidade: processos de validação. Tese de doutoramento - Universidade NOVA de Lisboa, Lisboa, 2014.

SOUZA, R. R.; TUDHOPE, D.; ALMEIDA, M. B. Towards a taxonomy of KOS: Dimensions for Classifying Knowledge Organization Systems. Knowledge Organization, v. 39, n. 3, p. 179192, jan. 2012.

TEMMERMAN, R. Towards new ways of terminology description: the sociocognitive approach. Amsterdam: John Benjamins, 2000.

WIERZBICKA, A. Semantics: primes and universals. Oxford: Oxford University Press, 1996.

WÜSTER, E. Introduction to the general theory of terminology and terminological lexicography. Vienna: Springer, 1979.

ZENG, M. Knowledge Organization Systems (KOS). Knowledge Organization, v. 35, n. 2/3, p. 160-182, 2008.

Recebido: 07/07/2021.

Aprovado: 23/07/2021. 